\title{
Evidence for explosive event activity originating in the chromosphere
}

\author{
J. G. Doyle ${ }^{1}$, B. Ishak ${ }^{1}$, I. Ugarte-Urra ${ }^{1, \star}$, P. Bryans ${ }^{2}$, and H. P. Summers ${ }^{2}$ \\ 1 Armagh Observatory, College Hill, Armagh BT61 9DG, N. Ireland, UK \\ e-mail: jgd@star.arm.ac.uk \\ ${ }^{2}$ Department of Physics, University of Strathclyde, Glasgow, Scotland, UK
}

Received 21 March 2005 / Accepted 9 May 2005

\begin{abstract}
We report on a joint SUMER, CDS, TRACE study, concentrating on a region which shows prolonged EUV explosive event (EE) activity in the transition region line N v $1238 \AA$, yet little evidence of such activity in another transition region line O V $629 \AA$ (formed at a similar temperature) which was observed simultaneously. A possible explanation for the lack of major activity in the $\mathrm{O} v$ line in several explosive events could be that they originate in the lower chromosphere. This is consistent with the enhancements in the C I $1249 \AA$ line and with the findings of another study which reported time delays between the chromospheric and transition region lines in some EE's using high cadence observations (10 s exposure time) obtained with the SUMER spectrometer in H I Ly $6(20000 \mathrm{~K})$ and S VI $(200000 \mathrm{~K})$. Using the generalized collisional-radiative picture, including the population of metastable levels, we derive the density dependent contribution function for both N V 1238 and O v 629 for four values of the electron density; $10^{6} \mathrm{~cm}^{-3}$ representing the low density limit, $10^{9} \mathrm{~cm}^{-3}$ for a typical quiet Sun electron density plus $10^{11} \mathrm{~cm}^{-3}$ and $10^{12} \mathrm{~cm}^{-3}$ for an active region. These calculations show that with increasing electron density, both lines shift to slightly lower temperatures. However, the major difference is in the relative increase in the line flux with increasing density. For $\mathrm{N} \mathrm{v}$, increasing the density to $10^{11} \mathrm{~cm}^{-3}$ results in a $60 \%$ increase in the line flux, while $\mathrm{O} \mathrm{v}$ shows a $30 \%$ decrease. Increasing the electron density to $10^{12} \mathrm{~cm}^{-3}$ results in a factor of two decrease in the $\mathrm{O} v$ flux, thus making it difficult to detect explosive event activity in this line if the event is formed in the chromosphere. Other explosive events which show simultaneous activity in both lines are probably formed in the transition region. In one such event, activity is observed in both $\mathrm{N} \mathrm{V}$ and $\mathrm{O}$ V, yet nothing in $\mathrm{C}$ I. In this event we also observe an increase in the TRACE 173 emission, delayed by $\approx 40 \mathrm{~s}$ compared to the transition region lines.
\end{abstract}

Key words. Sun: corona - Sun: transition region - Sun: chromosphere - line: formation - atomic processes - Sun: activity

\section{Introduction}

High resolution spectral and spatial coverage of the Sun over the last decade from a range of telescopes aboard many spacecraft has led to a wealth of observations of small-scale dynamic events observed from the chromosphere to the transition region and corona. One of the most observed but yet not understood are explosive events (EE's), also termed bi-directional jets. They were first discovered and classified as turbulent events by Brueckner \& Bartoe (1983). They are characterized by highly non-Gaussian profiles due to an enhancement in the blue and red wings. Most of the events are predominantly blue-shifted, showing Doppler shifts up to $150 \mathrm{~km} \mathrm{~s}^{-1}$. They appear preferably in regions with weak fluxes of mixed polarity or on the border of regions with large concentration of magnetic flux (Chae et al. 1998). Their average lifetime ranges from $\sim 60$ to $350 \mathrm{~s}$. In an analysis of a large raster, Teriaca et al. (2004) estimated an average size of $1800 \mathrm{~km}$ and a birthrate of $2500 \mathrm{~s}^{-1}$

^ Now at: Naval Research Laboratory, Code 7673U, 4555 Overlook Ave SW, Washington DC 20375, USA. over the entire Sun. They are often observed in bursts lasting up to $30 \mathrm{~min}$ in regions undergoing magnetic cancellation (Dere 1994).

Time delays between the chromospheric and transition region lines have being investigated (Madjarska \& Doyle 2002) using high cadence observations (10 s exposure time) obtained with the SUMER spectrometer in H I Ly $6(20000 \mathrm{~K})$ and S VI $(200000 \mathrm{~K})$. A time delay in the response of the $\mathrm{S}$ VI line with respect to the H I Ly 6 line was observed. This suggests that the process which generates these jets first occurs in the low chromosphere although they are best observed in transition region lines.

Teriaca et al. (2001) obtained high spectral resolution data for a large event in the transition region line N V $1238 \AA$ and the coronal line Mg X $625 \AA$. They concluded that the event observed in $\mathrm{N} V$ showed a small enhancement in $\mathrm{Mg} \mathrm{X}$ which was however due to the presence of a close-by blend from a $\mathrm{Si}$ II line. The conclusion was that EUV bi-directional jets are not directly relevant in heating the corona and are characteristic of structures not obviously connected with the upper corona. 
Table 1. A summary of the CDS data set consists of two rasters (I and III) and a time series (II), taken on 1999 June 1.

\begin{tabular}{ccccccc}
\hline \hline & Data & Observation & Start time & Exp. time & Duration & Coordinates \\
\hline I & s16287 & AR_MON/v54 & $07: 56 U T$ & $25 \mathrm{~s}$ & $55 \mathrm{~m} 53 \mathrm{~s}$ & $(394,385)$ \\
II & s16288-98 & MOSS_1M/v1 & $09: 01 \mathrm{UT}$ & $25 \mathrm{~s}$ & $03 \mathrm{~h} 12 \mathrm{~m} 32 \mathrm{~s}$ & $(401,405)$ \\
III & s16299 & AR_MON/v54 & $12: 14 \mathrm{UT}$ & $25 \mathrm{~s}$ & $55 \mathrm{~m} 53 \mathrm{~s}$ & $(423,384)$ \\
\hline
\end{tabular}

In some instances, a non-Maxwellian electron distribution may be present (Doyle et al. 2003). With a non-Maxwellian electron distribution, a significant fraction of the emission typical for a plasma at a temperature just below $1 \mathrm{MK}$ can instead originate at temperatures around $300000 \mathrm{~K}$.

Here, we analyse part of a joint SUMER, CDS and TRACE study, concentrating on a region which shows prolonged EE activity in the transition region line N V $1238 \AA$, yet little evidence of such activity in another transition region line O v $629 \AA$ observed simultaneously.

\section{Observational data}

\subsection{SUMER}

The Solar Ultraviolet Measurements of Emitted Radiation (SUMER) spectrograph (Wilhelm et al. 1995; Lemaire et al. 1997) onboard Solar Heliospheric Observatory (SoHO) was designed to provide line profile measurements at both high spatial and spectral resolution as well as a wide spectral coverage ranging from less than 500 to $1610 \AA$.

The data set used here was taken on 1999 June 1 starting at 09:13 UT and ending at 11:01 UT. Slit $0.3^{\prime \prime} \times 120^{\prime \prime}$ was used, exposing for $25 \mathrm{~s}$ on the bottom part of detector B for Mg X $624.95 \AA$ (in second order), O v $629.73 \AA$ (in second order), N v $1238.82 \AA$, N v $1242.80 \AA$, C I $1249 \AA$ and Si II $1251.16 \AA$ lines. The temporal sequence consists of spectral windows of 120 spatial $\times 50$ spectral pixels. The solar rotation compensation mode was turned on for these observations.

The $\mathrm{O} v$ line was observed in second order at a wavelength of $1259.52 \AA$, based on Si I $1258.80 \AA$ and Si II $1260.422 \AA$ (W. Curdt, private comm.) as a chromospheric reference. It is therefore blended with S II 1259.51. We can use two methods to estimate the strength of the blended S II line. From the quiet sun atlas of Curdt et al. (2001) we can get the strengths of the two S II lines at $1253.813 \AA$ and $1250.587 \AA$, then using CHIANTI (Young et al. 2003) one can derive the relative strength of the line at 1259.51. Since O V was observed on the bare part of the photocathode, the contribution from the S II blend is insignificant in this instance. Alternatively, SUMER allows decomposition of blends, if lines are observed in different orders. Thus an estimate of the S II blend in first order can be calculated from the data set used for the QS atlas by Curdt et al. (2001) using both the $\mathrm{KBr}$ and bare exposures. The measured counts are linear combinations of the contributors $\mathrm{O}$ V and S II (W. Curdt, private comm.) involving the coefficients of well-defined sensitivity values and photon fluxes. Solving the linear equation, yields the photon fluxes. In the Curdt et al. (2001) quiet sun atlas, the contribution of S II counts was $<5 \%$ on $\mathrm{KBr}$, and therefore on bare even less.
SUMER data reduction involves several steps. We decompressed and reversed the raw data, applying a flat field correction to correct the non-uniformities in the sensitivity of the detector. A geometrical distortion correction was also applied so that the rest position of the line profiles is on the correct spectral pixel and the slit images are straightened. To further analyze the data set, we have used a Gaussian-fitting procedure in the SolarSoft $(\mathrm{SSW})^{1}$ library to get the amplitude, central position, full width at half maximum (FWHM) and $\chi^{2}$ of the line profiles. Due to instrumental broadening, the FWHM needs to be corrected by applying the con_width_funct_3.pro routine. The $\chi^{2}$ values are used to check the goodness of the fit. The total intensity of the lines can be derived from the peak amplitude and FWHM.

\section{2. $C D S$}

The Coronal Diagnostic Spectometer (CDS; Harrison et al. 1995) also onboard SoHO, was designed to obtain spectroheliograms in a number of lines simultaneously in the extreme ultraviolet (EUV) region of the electromagnetic spectrum. CDS has two spectrometers, namely, the Grazing Incidence Spectrometer (GIS) and Normal Incidence Spectrometer (NIS). Our data set, observed on 1999 June 1 was obtained with NIS.

Prior to and after the time sequence, $244^{\prime \prime} \times 240^{\prime \prime}$ rasters using the $4^{\prime \prime} \times 240^{\prime \prime}$ slit were obtained. A time series using the $2^{\prime \prime} \times 240^{\prime \prime}$ slit with a cadence of $31 \mathrm{~s}$ was taken from 09:01 UT to 12:13 UT. Unfortunately, the CDS time series are offset with respect to the SUMER pointing by more than ten arcseconds. Only the O v $629.73 \AA$ context rasters were therefore used in our study. The standard CDS data reduction routines were applied to clean the data of cosmic rays, debias, flat-field and to correct the offset between NIS1 and NIS2. Details of the observations are given in Table 1.

\subsection{TRACE}

Transition Region and Coronal Explorer (TRACE; Handy et al. 1999), is a NASA Small Explorer (SMEX) mission devoted to studying the evolution and propagation of fine-scale magnetic fields and plasma structures throughout the solar atmosphere. TRACE consists of a telescope with a $30 \mathrm{~cm}$ primary mirror, normal incidence coatings for three EUV bands (173, 195 and $284 \AA$ ), and interference filters for UV bands (1216 to $1700 \AA$ ) as well as white light (temperature ranges from $\sim 6000$ to $\sim 2.5 \times 10^{6} \mathrm{~K}$ ). The $1024 \times 1024 \mathrm{CCD}$ camera has a field of view of $8.5^{\prime} \times 8.5^{\prime}$ with a spatial resolution of $1^{\prime \prime}$ and exposure times of 0.002 to $260 \mathrm{~s}$ with a cadence as short as $2 \mathrm{~s}$.

\footnotetext{
${ }^{1}$ http://www.lmsal.com/solarsoft/.
} 

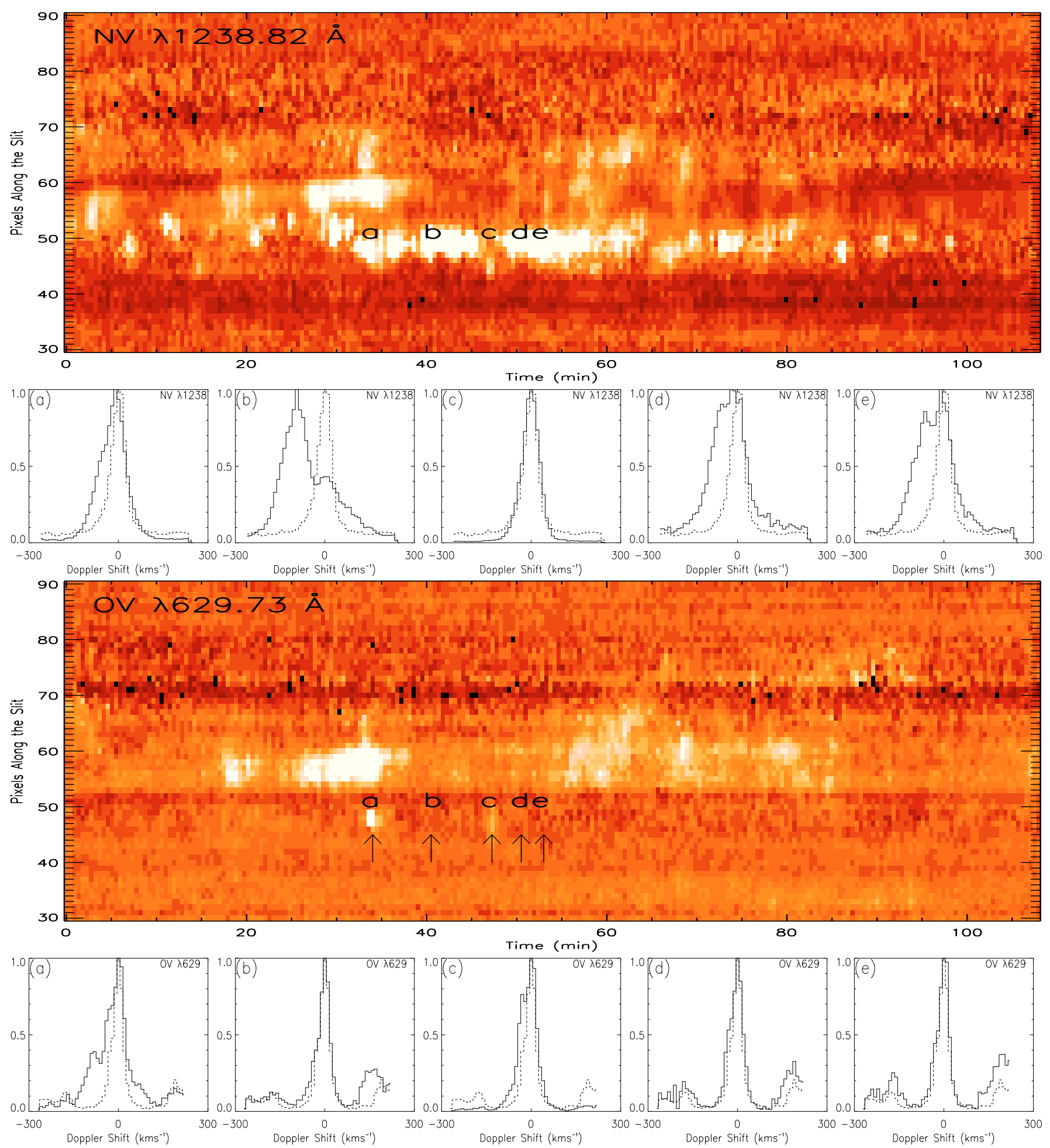

Fig. 1. Time series image plots for N v 1238 (top) O v 629 (bottom) over the whole duration of the dataset showing the variation in the line width assuming a single Gaussian fit. The data set was taken on 1999 June 1 starting at 09:13 UT. Below each image we show the resulting line profile for both $\mathrm{O} v$ and $\mathrm{N} v$ taken at the same time at five different temporal locations (09:47, 09:53, 10:00, 10:03 and 10:05 UT) labeled a)-e), normalized to unity. The quiet Sun profile is over-plotted for comparison (dashed line).

TRACE 173 Å images discussed here were obtained starting at 09:02 UT and finishing at 12:00 UT on 1999 June 1. The integration time of each image was 5.8 s from 09:02 UT until 09:41 UT and 4.1 s after that time. Images were taken every $12 \mathrm{~s}$. We discuss here only the data which were obtained simultaneously with the SUMER spectrometer. In addition, white-light images were obtained every 12 min starting at 09:22 UT. The instrument was pointed at coordinates Solar_X $=422^{\prime \prime}$ and Solar_Y $=401^{\prime \prime}$. 


\section{4. $M D I$}

Only three full-disk MDI (Michelson Doppler Interferometer, Scherrer et al. 1995) magnetograms were available for the duration and pointing coordinates of the observations. White light images were used to co-align TRACE and MDI. The magnetograms show that the EE appear in a region of mixed polarity weak fields. The flux densities are close or below the noise level of the magnetograms (12 $\mathrm{Mx} \mathrm{cm}^{-1}$; see Hagenaar 2001 for estimation method).

\subsection{Alignment}

Two steps were required to determine the position of the SUMER slit in the TRACE field of view. Firstly, the 08:32 UT TRACE $173 \AA$ image was co-registered to the Mg IX $368 \AA$ CDS context raster taken in the interval 07:56-08:52 UT, via cross-correlation. Similarly, that technique was used to find the match of the O v $629 \AA$ intensity profile along the SUMER slit (in its original form and degraded to the CDS spatial resolution) in one of the South-North cross-sections of the O V CDS context raster. The location of the SUMER slit in the TRACE images was inferred from the comparison. Similar steps were used to determine that the slit in the CDS time series is off-set with respect to the SUMER pointing.

\section{Explosive event identification}

As noted by many investigators, quiet-Sun profiles are generally well represented by a single Gaussian. In an analysis of an O VI $1032 \AA$ raster, Teriaca et al. (2004) noted that an excellent way to locate EE's was to check the locations where one of the fitted parameters deviated by more than $3 \sigma$ from the average of its distribution. In Fig. 1, we show a section of the O V $629 \AA$ and N V $1238 \AA$ time series, where the images shown are derived from the line width. The enhanced regions are those with larger widths, i.e. those that we assigned a preliminary flag as being possible EE's. The individual profiles were then checked to see whether there was evidence of a high velocity explosive component.

\section{Results}

For illustrative purposes, we show five temporal locations, indicated by the letters (a), (b), (c), (d) and (e) for summed pixels 47 to 50 along the slit direction. In the $\mathrm{N} \mathrm{V}$ profiles, these times indicated EE activity, with several of them indicating mass flows in excess of $150 \mathrm{~km} \mathrm{~s}^{-1}$ and line intensity increases sometimes exceeding the normal "quiet" Sun component. With exception of event (a), the $\mathrm{O} v$ line profiles fail to show similar evidence of large-scale EE activity at some locations. Instead, we see either none or in some instances, only a minor indication of mass flows (see Fig. 2). Here we show light-curves for the three components of both lines: blue, main and red. The main component results from summing over 9 spectral pixels, i.e. $\pm 45 \mathrm{~km} \mathrm{~s}^{-1}$ for $\mathrm{N} \mathrm{v}$ and half this value for $\mathrm{O} \mathrm{v}$, with the blue-wing and red-wing components extending from these values out to $\approx 160 \mathrm{~km} \mathrm{~s}^{-1}$ and excluding the chromospheric
$\mathrm{Si}$ II line to the red of the $\mathrm{O} \mathrm{V}$. In addition, the corresponding TRACE $173 \AA$ A light-curve is given, plus the weak chromospheric C I 1249 line.

It is at first hard to understand the lack of similar activity in both lines as they are considered to be formed at a similar temperature. For example, using CHIANTI, the peak formation temperature of $\mathrm{N} \mathrm{V}$ is $200000 \mathrm{~K}$ while $\mathrm{O} \mathrm{V}$ has a peak formation temperature of $250000 \mathrm{~K}$, considering the ionization balance calculations of Mazzotta et al. (1998).

Several other EE's were detected during the $\approx 100 \mathrm{~min}$ of observations at different locations along the slit, e.g. between pixels 57 and 60 along the slit around 09:35 UT. Here, both the $\mathrm{N} \mathrm{V}$ and $\mathrm{O} \mathrm{V}$ lines show the presence of prolonged EE activity. The largest of the $\mathrm{N} v$ EE's is at location (b), with the event showing a peak counts of close to $500 \mathrm{cts} /$ pixel in the blue-shifted plasma compared to the quiet Sun counts of $130 \mathrm{cts} /$ pixel in the stationary component. This event is shown in more detail in Fig. 3. The peak count rate in the $\mathrm{O} \mathrm{V}$ line is similar in both the quiet sun and EE (b).

\section{Discussion}

In an effort to try to understand how the EE's are easier identified in the $\mathrm{N} \mathrm{V}$ line, while they are practically absent in $\mathrm{O} \mathrm{V}$ despite the fact that both of these lines are formed at very similar temperatures, we look closely at their formation. The usual practice when considering atomic processes in hightemperature, low-density plasmas, such as discussed here, is to adopt the coronal approximation. This treats the populations of excited states of ions via an excitation balance of collisional excitation, usually from the ground state, by electrons, and radiative decay. The ionization state is established as a balance of electron impact ionization from the ground state and radiative plus dielectronic recombination. In the simplest version of such modeling, secondary collisions with excited states are neglected. However, there is no consistent treatment of metastable states with populations comparable to the ground state. Thus, in the coronal limit, ionization and excitation balance are independent of electron density. Recent work by Doyle et al. (2005) has looked at this problem regarding formation of the Li-like lines only. Here, we have a line from a Li-like ion and the other is from a Be-like ion.

The generalized collisional-radiative picture (Summers \& Hooper 1983), which builds on the collisional-radiative theory of Bates et al. (1962), allows such an analysis. In detail, the collisional ionization and redistribution processes from excited states, ignored in the coronal model, are included. The populated metastable states are determined via an elaborated ionization balance along with the ground states. These were computed within the Atomic Data and Analysis Structure (ADAS; Summers $2004^{2}$ ) framework, which is a collection of fundamental and derived atomic data, and codes that manipulate them. The data are organized in a form that allows generation of the collisional-radiative matrix, which is manipulated to form the derived effective recombination and ionization coefficients. There is no significantly populated metastable level

\footnotetext{
${ }^{2}$ http://adas.phys.strath.ac.uk
} 

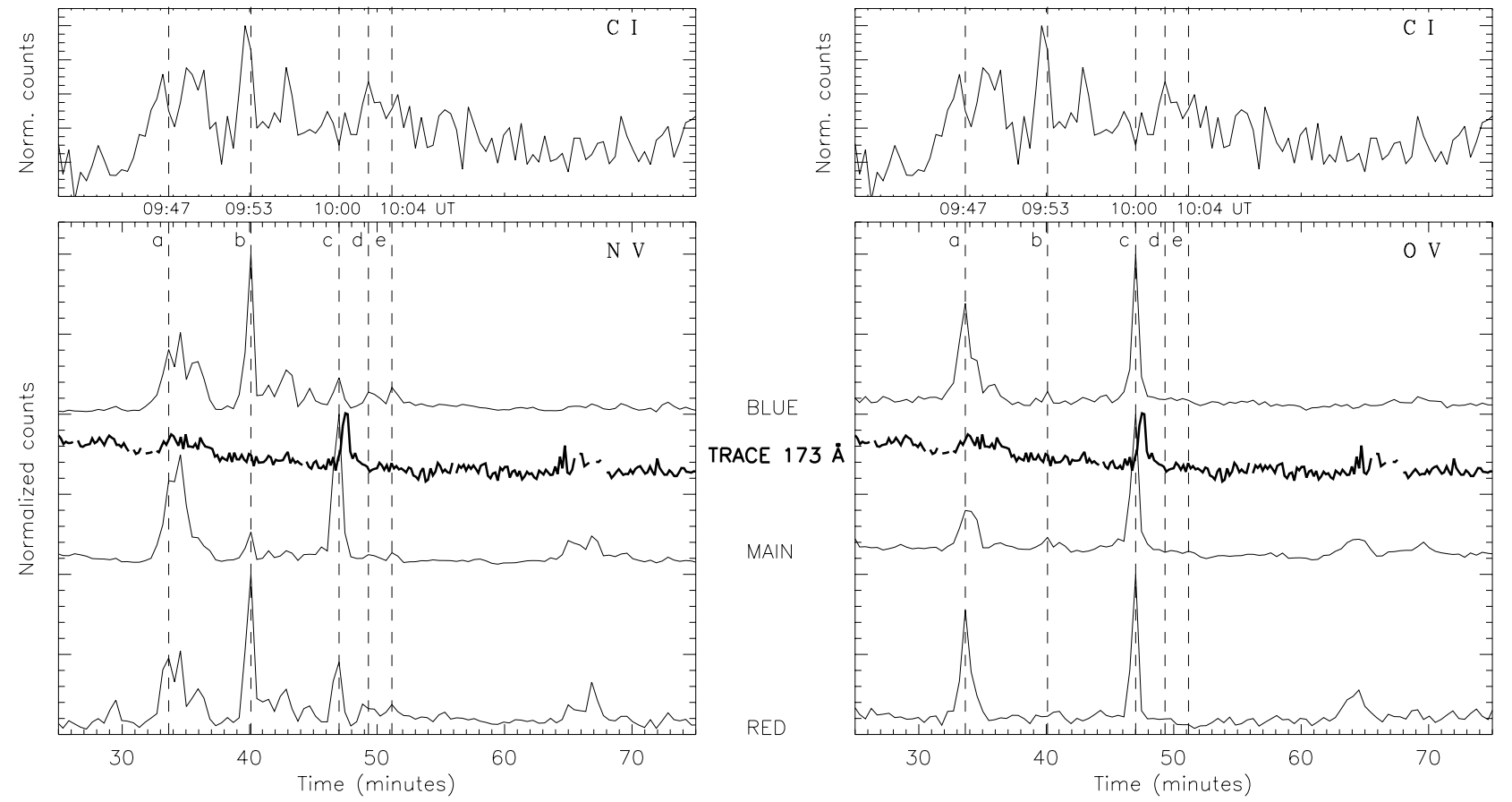

Fig. 2. Normalized light-curves for N V 1238 and O v 629 for the blue wing, red wing and main component, plus the corresponding TRACE $173 \AA$ band and the weak C I 1249 line. The gaps in the TRACE light curve are missing data due to the presence of cosmic rays.
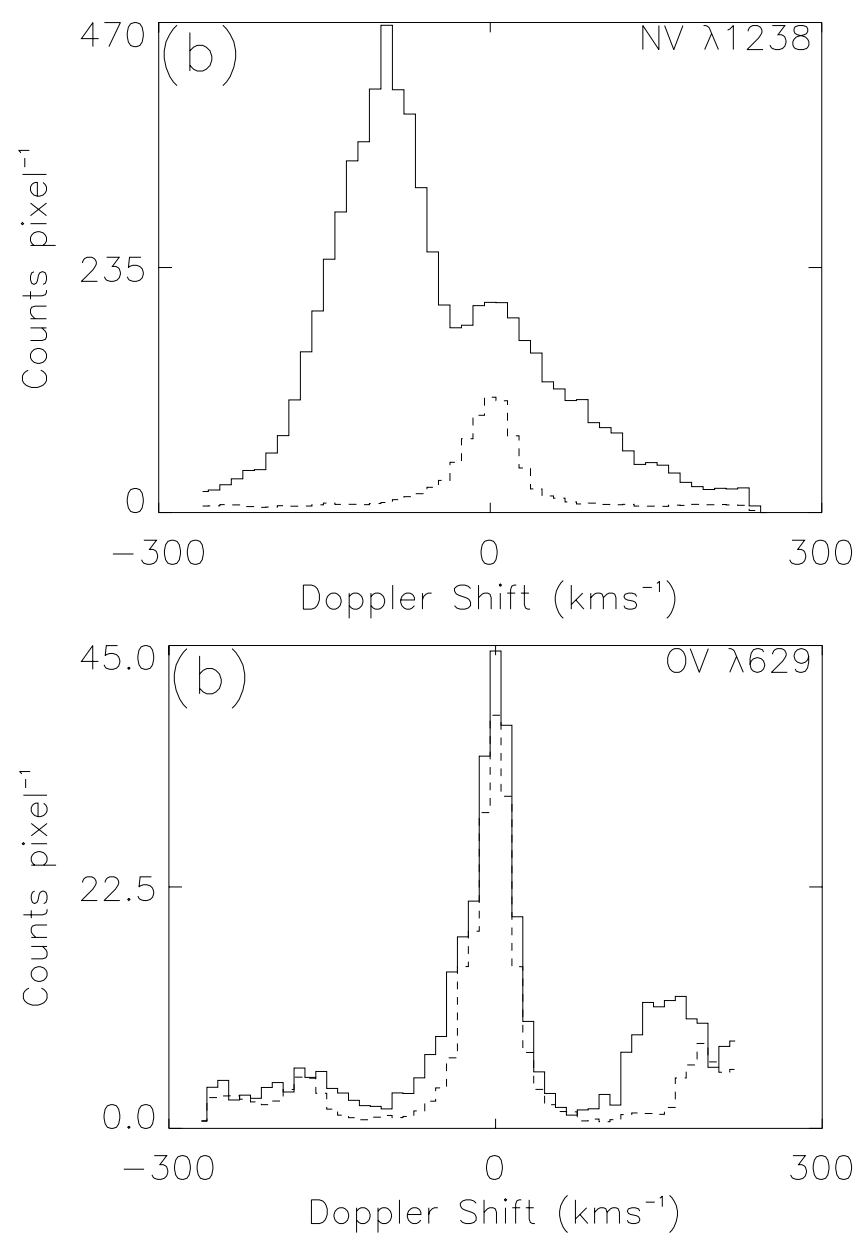

Fig. 3. The $\mathrm{O} v$ and $\mathrm{N} v$ line profile at 09:53 UT, i.e. event b). The quiet Sun profile is over-plotted for comparison (dashed line).

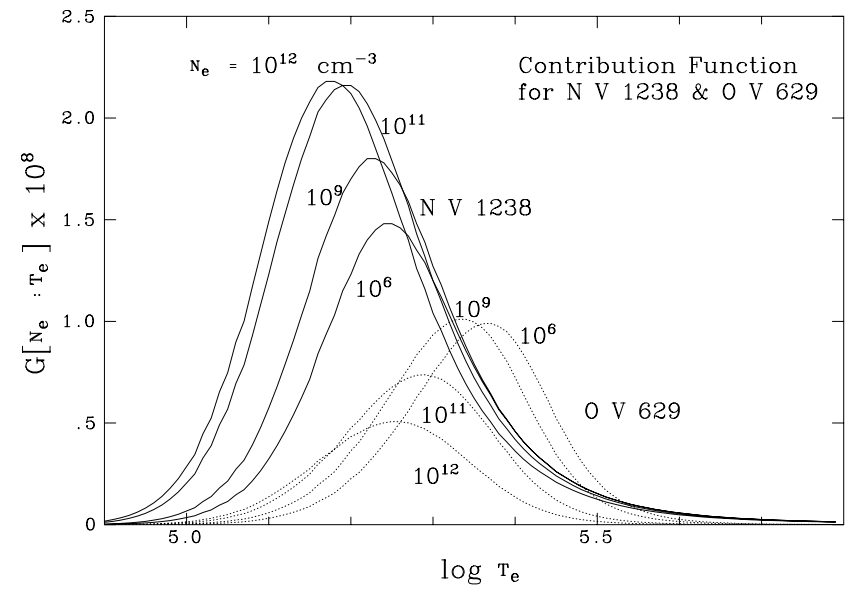

Fig. 4. The density dependent contribution function for O V $629 \AA$ and N v $1238 \AA$ for four values of the electron density, $N_{\mathrm{e}}=10^{6} \mathrm{~cm}^{-3}$, $10^{9} \mathrm{~cm}^{-3}, 10^{11} \mathrm{~cm}^{-3}$ and $10^{12} \mathrm{~cm}^{-3}$.

in the case of Li-like ions, but the metastable resolved picture matters when considering recombination and ionization to and from the He-like and Be-like stages. Further details are given in Doyle et al. (2005).

In Fig. 4 we show the density dependent contribution function for $\mathrm{N} \mathrm{V} 1238$ and O V 629 for four values of the electron density; $10^{6} \mathrm{~cm}^{-3}$ representing the low density limit, $10^{9} \mathrm{~cm}^{-3}$ for a typical quiet Sun electron density, plus $10^{11} \mathrm{~cm}^{-3}$ and $10^{12} \mathrm{~cm}^{-3}$ for an active region. Here we see that with increasing electron density, both lines shift to slightly lower temperatures. However, the major difference is in the relative increases in the line flux with increasing density. For $\mathrm{N} \mathrm{v}$, increasing the density to $10^{11} \mathrm{~cm}^{-3}$ results in a $60 \%$ increase in the line flux, while $\mathrm{O} v$ shows a $30 \%$ decrease. Increasing the 

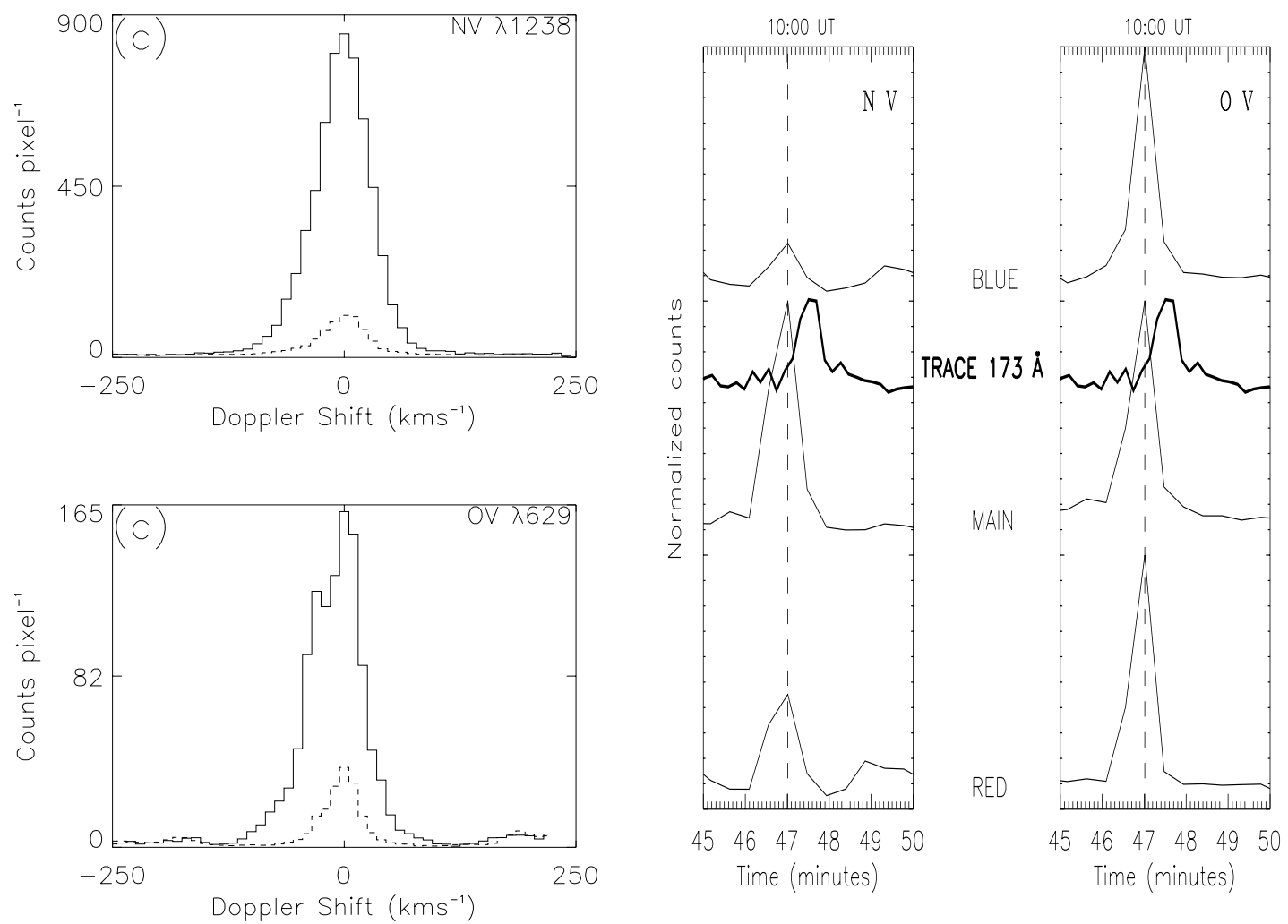

Fig. 5. Left: the O V and N v line profiles at 10:00 UT, i.e. event c). The quiet Sun profile is over-plotted for comparison (dashed line). Right: close-up of the time series shown in Fig. 2 corresponding to the interval 45-50 min.

electron density to $10^{12} \mathrm{~cm}^{-3}$ results in a factor of two decrease in the $\mathrm{O} v$ flux. This decrease in the line flux can be explained by considering the relative populations of the metastable and ground terms of the $\mathrm{O}^{+4}$ ion. At low densities the metastable population is negligible but, on increasing the density, the population becomes significant and acts as a sink to higher levels, thus causing the $629 \AA$ transition to be depleted.

Hence, a possible explanation for the lack of major activity in the $\mathrm{O} v$ line for events (b), (d) and (e) could be that these EE's occur in the lower chromosphere, thereafter in a high electron density region. Since EE's are predominately line-shift events as opposed to line flux enhanced events, the increased sensitivity of $\mathrm{N} \mathrm{V}$ by a factor of two compared to $\mathrm{O} \mathrm{V}$ which is decreased by a factor two, means that these EE's are less visible in $\mathrm{O} \mathrm{V}$.

This is consistent with the findings of Madjarska \& Doyle (2002) who found time delays between the chromospheric and transition region lines in some EE's using high cadence observations (10 s exposure time) obtained with the SUMER spectrometer in H I Ly $6(20000 \mathrm{~K})$ and S VI $(200000 \mathrm{~K})$. This suggested that the process which generates these jets first occurs in the low chromosphere although they are best observed in transition region lines.

In another study of EE's, Doyle et al. (2003) used a nonMaxwellian electron distribution to explain how "coronal" plasma as detected in the TRACE imager with the $173 \AA$ filter could derive from temperatures around $\approx 300000 \mathrm{~K}$, as opposed to $\approx 800000 \mathrm{~K}$. These authors had an example of a bi-directional jet registered in the chromospheric and the transition region lines but not showing any detectable signature in the coronal line, yet was clearly detected in the TRACE $173 \AA$ pass-band. Hence, it is possible that these transition region lines could be effected by a non-Maxwellian electron distribution, thus emitting at close to chromospheric temperatures. However, we rule this out as a possible explanation for the lack of activity in events (b), (d) and (e), although this may not be the case for event (c). In Fig. 5 we show the $\mathrm{N} v$ and $\mathrm{O} v$ line profiles, in addition to an expanded plot of the various lightcurves for this event.

Figure 2 shows that this event is observable in both $\mathrm{O} \mathrm{V}$ and $\mathrm{N} \mathrm{V}$, in addition to TRACE, thus perhaps questioning the increased electron density suggestion. However, a close inspection of Fig. 1 shows that part of this event is shifted a few arcsecs from the other events and thus is likely to be occurring in different plasma conditions than the others.

Further evidence for the chromospheric nature of events (b), (d) and (e) comes from the observed increase in the Si II 1251 line to the red of O V, see Figs. 1, 3 and 5. In this low temperature line, although we only observe the redwing, a large flux increase is apparent. However, for event (c) (see Fig. 5) the Si II line has a similar intensity in both the quiet Sun and during the EE. This would suggest that unlike the other events, this EE is produced higher in the atmosphere, probably in the transition region at around a temperature of $250000 \mathrm{~K}$. 
Furthermore, as shown in Fig. 2, the C I line shows an increase in intensity for events, (a), (b), (d) and (e), consistent with the idea that these EE's are produced in a low temperature plasma, but no apparent increase is seen for event (c). For event (c), the $\mathrm{N} \mathrm{v}$ line shows very broad profiles over two consecutive exposures with only minimum evidence for secondary components. The $\mathrm{O} \mathrm{V}$ on the-other-hand shows a secondary blueshifted component, with broadened profiles stretching up to $5^{\prime \prime}$ to the north. The TRACE 173 light-curve peaking some $40 \mathrm{~s}$ after the rise in $\mathrm{N} \mathrm{V}$ and $\mathrm{O} \mathrm{V}$ may very well be coronal in origin. To test this idea further, we looked closely at the $\mathrm{Mg}$ × 625 in second order and the weak C I 1249 line.

The Mg X $625 \AA$ line is observed as a second order line at $1249.90 \AA$, therefore, it overlaps with several first order chromospheric lines, namely P II 1249.82, Mg II 1249.93 and Si II 1250.09. A detailed discussion on these blends is given by Teriaca et al. (2001) and Doyle et al. (2003). The latter authors performed a detailed spectroscopic analysis for the largest of these EE's (i.e. event b) in order to estimate the contribution of the spectral lines blending to $\mathrm{Mg} \mathrm{X}$ when recorded on the $\mathrm{KBr}$ part of detector $\mathrm{B}$. The average contribution for the Si II $1250.09 \AA$ line was found to be between 35 and $40 \%$. Another few percent can come from the $\mathrm{Mg}$ II and the P II lines leading to a total first-order contribution of $\approx 40 \%$. The result was that the small increase observed in $\mathrm{Mg} \mathrm{X}$ was due to the chromospheric contribution and thus the small observed increase in TRACE 173 did not come from the corona. These authors suggested the presence of a non-Maxwellian electron distribution to explain the TRACE data. Although in event (c) we have a much larger intensity increase in the TRACE 173 passband, as in previous studies by Teriaca et al. (2001) and Doyle et al. (2003), there is no apparent increase in the $\mathrm{Mg} \mathrm{X}$ line, suggesting either that the TRACE 173 increase is not from the coronal region or that the EE jet manifests itself as a flux increase at coronal temperatures as opposed to a velocity feature.

The above findings therefore suggest two types of EE's; one formed in the low chromosphere and the other formed in the mid-to-high transition region. This therefore suggests that single temperature line profile information is not sufficient to clarify the nature of these events. Furthermore, this again shows the importance of considering a full atomic model as opposed to the commonly adopted assumption of ionization and recombination to the ground-state only.
Acknowledgements. We would like to thank the SUMER team at Max-Planck-Institute for Solar System Research (MPS), and CDS and TRACE teams at Goddard Space Flight Center for their help in obtaining the present data. SUMER and CDS are part of SoHO, the Solar and Heliospheric Observatory, a mission of international cooperation between ESA and NASA. Research at Armagh Observatory is grant-aided by the N. Ireland Dept. of Culture, Arts and Leisure. BI wishes to thank E. O'Shea for productive discussions, plus M. Madjarska and L. Xia for their help with the SUMER software, and W. Curdt for discussions on the wavelength calibration and line blending. This work was supported in part by a PRTLI research grant for Grid-enabled Computational Physics of Natural Phenomena (Cosmogrid). CHIANTI is a collaborative project involving the NRL (USA), RAL (UK), and the Universities of Florence (Italy) and Cambridge (UK)

\section{References}

Bates, D. R., Kingston, A. E., \& McWhirter, R. W. P. 1962, Proc. Roy. Soc., A267, 297

Brueckner, G. E., \& Bartoe, J.-D. F. 1983, ApJ, 272, 329

Chae, J., Wang, H., Lee, C.-Y., Goode, P. R., \& Schühle, U. 1998, ApJ, 497, L109

Curdt, W., Brekke, P., Feldman, U., et al. 2001, A\&A, 375, 591

Dere, K. P. 1994, Adv. Space Res., 14, 13

Doyle, J. G., Dzifćáková, E., \& Madjarska, M. S. 2003, Sol. Phys., 218,79

Doyle, J. G., Summers, H. P., \& Bryans, P. 2005, A\&A, 430, L29

Hagenaar, H. J. 2001, ApJ, 555, 448

Handy, B. N., Acton, L. W., Kankelborg, C. C., et al. 1999, Sol. Phys., 187,229

Harrison, R. A., Sawyer, E. C., Carter, M. K., et al. 1995, Sol. Phys., 162,233

Madjarska, M. S., \& Doyle, J. G. 2002, A\&A, 382, 319

Mazzotta, P., Mazzitelli, G., Colafranceso, S., \& Vittorio, N. 1998, A\&AS, 133, 403

Lemaire, P., Wilhelm, K., Curdt, W., et al. 1997, Sol. Phys., 170, 105

Scherrer, P. H., Bogart, R. S., Bush, R. I., et al. 1995, Sol. Phys., 162, 129

Summers, H. P., \& Hooper, M. B. 1983, Plasma Phys., 25, 1311

Teriaca, L., Madjarska, M. S., \& Doyle, J. G. 2001, Sol. Phys., 200, 91

Teriaca, L., Banerjee, D., Falchi, A., Doyle, J. G., \& Madjarska, M. S. 2004, A\&A, 427, 1065

Wilhelm, K., Curdt, W., Marsch, E., et al. 1995, Sol. Phys., 162, 189

Young, P. R., Del Zanna, G., Landi, E., et al. 2003, ApJS, 144, 135 